\title{
Rare left ventricular metastasis from uveal melanoma
}

\author{
Enrico Maria Zardi ${ }^{1}$, Giulio Cacioli ${ }^{1}$, Chiara Giorgi ${ }^{2}$, \\ Emanuele Paolo Rafano Carnà ${ }^{1}$, Domenico Maria Zardi ${ }^{3}$ \\ ${ }^{1}$ Hospitalist Service, Campus Bio-Medico University, Rome, Italy \\ ${ }^{2}$ Radiology Department, S. Maria della Misericordia Hospital, Urbino, Italy \\ ${ }^{3}$ Department of Cardiology, II School of Medicine, University La Sapienza, \\ Ospedale Sant'Andrea, Rome, Italy
}

A 60-year-old white woman was admitted to our hospital, October 2016 because of two episodes of hematemesis in the previous $48 \mathrm{~h}$, intensification of fatigue and lack of appetite. A review of the patient's history revealed that a stage IV right ocular melanoma had been diagnosed 7 years prior to admission. The patient underwent proton beam therapy and then first-line and second-line chemotherapy treatment. When the patient was admitted she was affected by multiple subcutaneous (Fig. 1A), pericardial (Fig. 1B, C), pulmonary and hepatic metastases. Subcutaneous metastases had been previously treated with electrochemotherapy. Blood analysis showed mild anemia (red blood cells $2.980 .000 \mu \mathrm{L}$, hemoglobin $9.7 \mathrm{~g} / \mathrm{dL}$ ) and an increased inflammatory pattern (white blood cells $12540 \mu \mathrm{L}$ procalcitonin $1.7 \mathrm{ng} / \mathrm{mL}$, C reactive protein $10 \mathrm{mg} / \mathrm{L}$ ) as observed in multiple metastasis [1]. Physical examination revealed pale skin color, increased liver consistency and volume; no other abnormalities were found, including cardiac auscultation. Electrocardiogram and hemogasanalysis were also normal. In order to rule out upper gastrointestinal bleeding, esophagogastroduodenoscopy was performed and showed no abnormalities. Given the suspicion of pulmonary embolism, due to advanced cancer and recurrent haemoptysis, we performed a trans-thoracic echocardiography that showed normal parameters (left ventricular diastolic and systolic diameter $44 \mathrm{~mm}$ and $29 \mathrm{~mm}$, septal thickness $9 \mathrm{~mm}$, posterior wall thickness $8 \mathrm{~mm}$, ejection fraction $58 \%$, left atrial area $14 \mathrm{~cm}^{2}$, right atrial area $12 \mathrm{~cm}^{2}$, right ventricular diastolic and systolic diameter $27 \mathrm{~mm}$ and $16 \mathrm{~mm}$, TAPSE $18 \mathrm{~mm}$ ) and there were no signs of right heart overload; unexpectedly, a pedunculated $15 \times 13 \times 14 \mathrm{~mm}$ mass was present, adherent to the endocardium, involving the lower third of both the interventricular septum and the left ventricle inferior wall (Fig. 1C, D). The mass was slightly hyperechoic and non-homogeneous with irregular margins (Fig. 1D). The color-Doppler did not show significant vascularization of the mass. No signs of patent foramen ovale were observed.

The case was then discussed by a multidisciplinary team including oncologists and cardiac surgeons. Considering the evidence of multiple metastatic disease, the poor prognosis and thus the unfeasibility of any surgical and medical treatment of the patient, it was decided to stop any further diagnostic and/or therapeutical procedure and to continue palliative cure. The patient was then discharged 5 days after admission.

Uveal melanoma is the most common primary intraocular malignancy; approximately 1500 cases are diagnosed in the United States each year, most commonly arising in the choroid followed by the ciliary body. The patient can be entirely asymptomatic and the tumour diagnosed only on routine ophthalmic screening [2]. The heart may be colonized by metastatic cells of uveal melanoma through the vascular system but often the diagnosis is missed over a lifetime [3]. It has been known since 1954 that the right side of the heart is more commonly involved by metastases, whereas only few of them have been reported in the left heart [3-5].

Address for correspondence: Enrico Maria Zardi, PhD, MD, Hospitalist Service, Campus Bio-Medico University, Alvaro del Portillo 200, 00128, Roma, Italy, e-mail: e.zardi@unicampus.it 


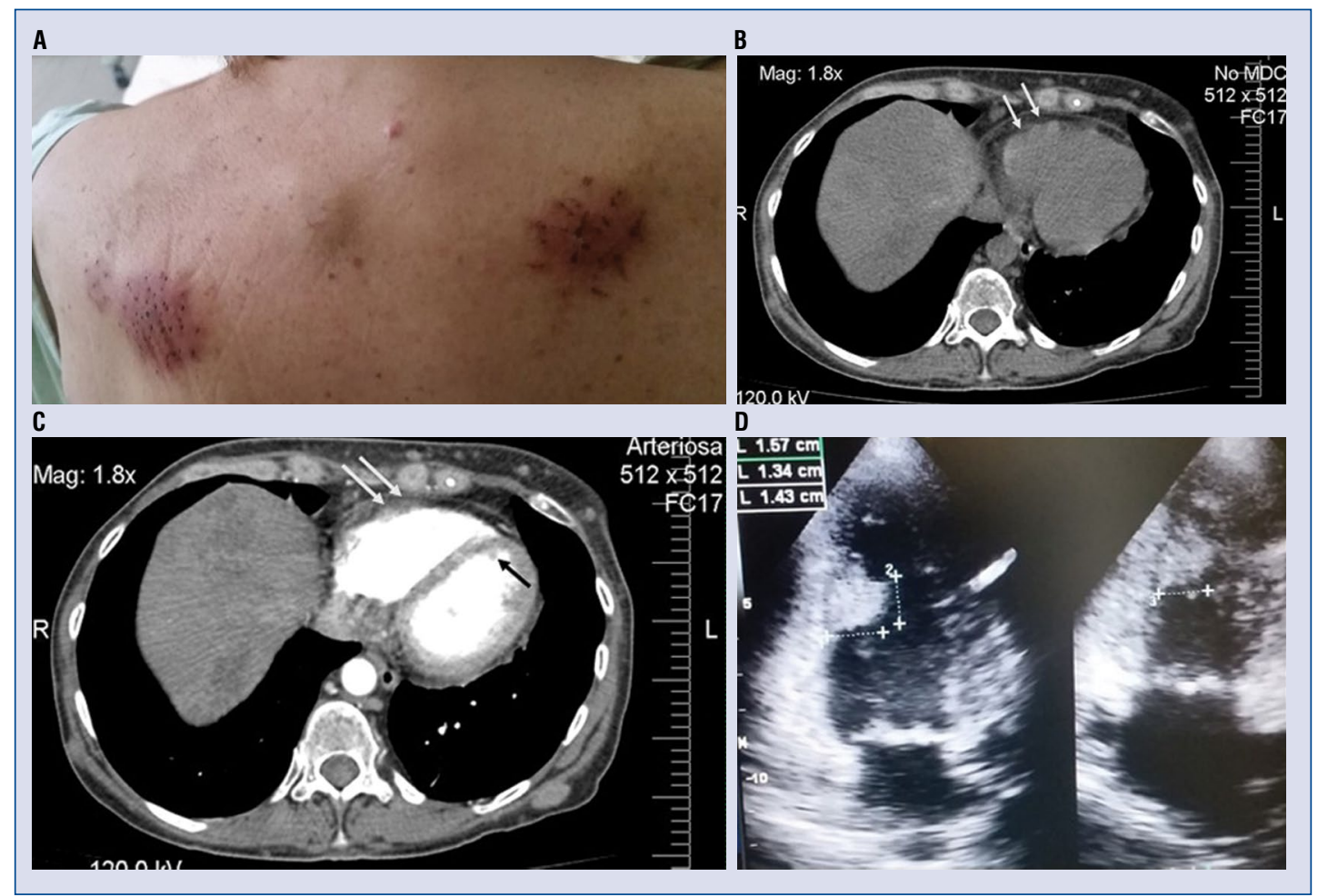

Figure 1. A. Interscapular region, subcutaneous metastases treated with electrochemotherapy; B, C. Unenhanced (panel B) and contrast-enhanced axial computed tomography (panel C) images through the heart showing focal thickening of the interventricular septum (black arrow) and nodular thickening of the pericardium (white arrows), representing metastases; D. Four (left) and two (right) chambers echocardiographic views showing hyperechoic and slightly non-homogeneous metastatic melanoma to the interventricular septum (calliper) and inferior free wall.

The evidence of a cardiac mass normally requires a complex differential diagnosis between benign tumors (myxoma, lipoma, papillary fibroelastoma, rhabdomyoma, fibroma, teratoma, Purkinje cells tumors/amarthomas, hemangioma), malignant tumors (angiosarcoma, rhabdomyosarcoma, fibrosarcoma, lymphosarcoma, osteosarcoma, liposarcoma), metastases, tumors that may be either benign or malignant (paraganglioma, mesothelioma) and non-neoplastic masses (intracardiac thrombus, endocarditis vegetation, lipomatous hypertrophy of interatrial septum, pericardial cyst, large coronary artery aneurism, crista terminalis) [6, 7]. However, due to the clinical history and condition of the patient the possibility excluded was that the mass at the left side of the interventricular septum was a benign tumor. Since the patient presented already pericardial metastatic localization of uveal melanoma and all other malignant tumors throughout the body had been excluded, it was logical to think that this intracardiac mass was metastatic and had spread from this tumor.

Cardiac metastases, when present, often represent a late manifestation of a neoplasm and should be suspected whenever a patient with known malignancy develops any cardiovascular symptoms or signs or an arrhythmia; even an embolism should raise the doubt of cardiac involvement in the hypothesis of cardiac origin. The specific symptoms would reflect the site of cardiac involvement.

As mentioned above, the occurrence of cardiac metastases is a possible but underdiagnosed localization in patients with melanoma when cardiac symptoms are missing as in our patient.

Since vascular spread appears as the most reasonable via of cardiac colonization for uveal melanoma, it would have been expected a localization of the mass in the right side of the heart. Indeed, lymphatic spread is not considered as a normal via through which metastatic cells may reach the interventricular left side of the heart. Therefore, a patent foramen ovale might be the via through which the metastatic spread occurred in our patient. When a tumor mass involves the left chambers, specialists should perform a transesophageal echocardiography to rule out a possible patent foramen ovale [8]. 
It was decided not to perform transesophageal echocardiography based on our evaluation of the absence of cardiovascular symptoms and poor prognosis.

Melanoma is a neoplasm with the highest propensity for metastasizing to the heart. Because patient signs and symptoms are often absent or nonspecific, clinical suspicion is paramount and echocardiography is the first line imaging modality. Cardiac computed tomography may confirm the presence of intracardiac masses [6].

In the case of this patient, based on clinical evaluation, transthoracic echocardiography and cardiac computed tomography, it was considered that the cardiac mass was a metastasis of advanced plurimetastatic uveal melanoma.

\section{Conflict of interest: None declared}

\section{References}

1. Avrillon V, Locatelli-Sanchez M, Folliet L, et al. Lung cancer may increase serum procalcitonin level. Infect Disord Drug Targets. 2015; 15(1): 57-63, indexed in Pubmed: 25809625.
2. Chattopadhyay C, Kim DW, Gombos DS, et al. Uveal melanoma: From diagnosis to treatment and the science in between. Cancer. 2016; 122(15): 2299-2312, doi: 10.1002/cncr.29727, indexed in Pubmed: 26991400.

3. Demondion P, Garot J, Bouvier E, et al. Left ventricular metastasis from ocular melanoma: a new, rare, and unusual pathway? Ann Thorac Surg. 2013; 96(1): e13-e14, doi: 10.1016/j.athoracsur.2012.12.019, indexed in Pubmed: 23816109.

4. Rosario RT, DiMaio DJ, Lapham RL, et al. Metastatic ocular melanoma to the left ventricle inducing near-syncope attacks in an 84-year-old woman. Chest. 2000; 118(2): 551-553, indexed in Pubmed: 10936157.

5. Ozyuncu N, Sahin M, Altin T, et al. Cardiac metastasis of malignant melanoma: a rare cause of complete atrioventricular block. Europace. 2006; 8(7): 545-548, doi: 10.1093/europace/eul058, indexed in Pubmed: 16798769.

6. Kassop D, Donovan MS, Cheezum MK, et al. Cardiac masses on cardiac CT: a review. Curr Cardiovasc Imaging Rep. 2014; 7: 9281, doi:10.1007/s12410-014-9281-1, indexed in Pubmed: 25018846 .

7. Goldberg AD, Blankstein R, Padera RF. Tumors metastatic to the heart. Circulation. 2013; 128(16): 1790-1794, doi: 10.1161/CIRCULATIONAHA.112.000790, indexed in Pubmed: 24126323.

8. Hara H, Virmani R, Ladich E, et al. Patent foramen ovale: current pathology, pathophysiology, and clinical status. J Am Coll Cardiol. 2005; 46(9): 1768-1776, doi: 10.1016/j.jacc.2005.08.038, indexed in Pubmed: 16256883. 